\title{
Mapping Meditative States and Stages with Electrophysiology: Concepts, Classifications, and Methods
}

\author{
Poppy L.A. Schoenberg ${ }^{1, *}$, David R. Vago ${ }^{1}$ \\ ${ }^{1}$ Contemplative Neuroscience and Integrative Medicine (CNIM) Laboratory, Vanderbilt University \\ *Corresponding author: 3401 West End Ave., Suite 380., Osher Center for Integrative Medicine, \\ Vanderbilt University Medical Center, Nashville, TN 37203. Email: poppy.schoenberg@ vanderbilt.edu. \\ Tel: +1 615-343-6991
}

Author Declarations: None we wish to confirm. There are no known conflicts of interest associated with this publication and there has been no significant financial support for this work that could have influenced its outcome. We confirm that the manuscript has been read and approved by all named authors and that there are no other persons who satisfied the criteria for authorship but are not listed. We confirm that we have given due consideration to the protection of intellectual property associated with this work and that there are no impediments to publication, including the timing of publication, with respect to intellectual property. In so doing We confirm that we have followed the regulations of our institutions concerning intellectual property. We understand that the Corresponding Author is the sole contact for the Editorial process (including Editorial Manager and direct communications with the office). We are responsible for submissions of revisions and final approval of proofs. We confirm that we have provided a current, correct email address which is accessible by the Corresponding Author. 


\begin{abstract}
Exploration of human consciousness remains a final frontier within basic neuroscience; that is, how the finite biological structure and function of the brain gives rise to the seemingly infinite expanse that encompasses the terrain of the mind. Contemporary mindfulness and other contemplative practices across historical and post-modern traditions involve systematic forms of mental training that allow the practitioner to develop the mind in very specific and quantifiable ways. Some fundamental questions pertain to this scientific enquiry; (1) how to concisely classify discrete and developmentally-specific "mind states" of consciousness that are in line with the subtle complex phenomenology of experience so to yield ontological quantifications? (2) what measures best represent such classification/quantification systems? (3) can the present electrophysiological purview map developmentally-specified mind states and stages to neurobiological substrates, based on extant contention (i.e. discrete EEG band functionality, phenomenological significance, and underlying mechanisms) regarding the interpretation of EEG physiology?
\end{abstract}

Keywords: meditation; EEG; consciousness; mindfulness; non-dual; insight 


\section{Introduction}

One empirical avenue to examine mind-states is through the lens of intentional structured mind training, such as meditation. Debate remains regarding a clear operational taxonomy of meditation for optimal utility within contemplative neuroscience (Nash \& Newberg 2013). Contemporary neuroscientific approaches to investigating meditation principally draw from a dual-band model defining two broad domains of meditative practice; focused attention (FA), and open monitoring (OM). A phenomenological matrix encompassing qualities of aperture, clarity, stability, and effort, pertaining to this FA-OM dualband model has been proposed (Lutz et al., 2015). Albeit, it could be reasoned that these phenomenological properties describe micro-level qualities of any given meditative state/practice. Moreover, preliminary evidence suggests that FA and OM are driven by discrete neuromodulator pathways. Phenotypal marker research identified through event-related potentials (ERPs) supports a potential role for attention-related neuromodulators in FA and OM regulation. Initial mindfulness-related evidence, from performance monitoring in attention-deficit/s, suggests that FA is primarily regulated by the dopaminergic system (and associated ERPs), wherein fixed-attentional focus activates and maintains activity in neural reward pathways. Conversely, OM practices are primarily driven by noradrenergic transmission (and associated ERPs), wherein a vigilance neural system facilitates reflexive 'switching' ability and more global attention/awareness, vigilance system (Schoenberg et al., 2014). Related ERP studies examining meditation support this hypothesis (Cheng et al., 2017; Sanger et al., 2016; Quaglia et al., 2015). Focus on ERP meditation correlates is relatively emerging however, since the mass of EEG studies have targeted oscillatory activity. Generally, findings highlight trends of modulated midline theta-, alpha-, and gamma-band frequencies, albeit direction of change pertaining to discrete bandwidths has not been consistent across studies (review Lee et al., 2018; DeLosAngeles et al., 2016; Kaur \& Singh, 2015; review Cahn \& Polich, 2013). We suggest that this convolution may be accounted for in part by differences in the micro-level qualities (clarity, stability, aperture, effort) within meditative state/s, and more largely by discrete macro-level phenomenology dependent upon developmental stages of progress, evident in mixed experiential descriptions from meditators. Ergo, what do we currently know about electroencephalographic (EEG) correlates of meditation, and how do they map onto stages of progress?

\section{EEG correlates of Meditation: Current Overview}

Consciousness is not a unitary concept, hence, quantification of brain-mind parameters involved during meditation is enriched from electrophysiological measurements. Technical advances of EEG and mathematical signal processing techniques enables the decomposition of non-stationary, non-linear, brain activity into temporal, frequency, phasic, and spatial, dimensions, that can be mapped onto, and represent the multi-dimensionality of, specific mind-states with ontological meaning for consciousness research. 
Albeit, extant proposals of neurophysiological/EEG meditation "signatures" is somewhat convoluted and lacking specificity (recent review, Lee et al., 2018). In part, this stems from: (i) a general trend to disproportionately examine one-dimension of the EEG signal, e.g. frequency spectra, with the aim to gauge meditation-associated changes in global and/or relative power spectra values, and (ii) ambiguity within the general EEG research field on the mechanisms/systems involved in EEG signal generation, neural computation, and representation. This is fundamentally problematic since a clear scientific understanding of what 'power-bands' represent, both functionally and phenomenologically, is not fully elucidated and must be interpreted within the context of the experimental design and elicitation pathways of the brain activity in question. Since these points are multi-faceted and beyond the scope of this commentary, a connected basic-science perspective can be found in Trends in Neuroscience (Cohen, 2017).

Despite the above barriers in identifying EEG correlates of meditation states/training, recent studies continue to design, rationalize, analyse, and interpret EEG data around rudimentary understandings of EEG oscillatory activity, that have in part emanated from clinical neurophysiology and neurofeedback applications where relative-based changes have clinical merit/meaning. From a basic-science perspective, typical frequency analyses during meditative blocks leads to over-generalized, theoretically and empirically gross-level conclusions, i.e. that a meditation technique changes theta/alpha/beta/gamma, evoked activity. The outcome of this rationale and approach in the extant literature yields EEG correlate patterns that are difficult to interpret with limited homogeneity in findings related to meditation, or even amongst the same meditation techniques (Lee et al., 2018; Kaur \& Singh, 2015). Recent meditation EEG studies continue to report EEG power band measures only (Korde \& Paikrao, 2018; Harne \& Hiwale, 2018; DeLosAngeles et al., 2016; Amihai \& Kozhevikov, 2014). Conversely, using the 'brain-as-radioreceiver/transmitter' metaphor, a multitude of frequencies (band-widths) are essentially (non-) localized simultaneously. One may conjecture that as a tuning function performs on a radio, certain brain circuits and synchrony relevant to the volitional state of specific meditative micro-level characteristics (e.g. stability, clarity) manifest distinct phenomenology. Such frequency-tuning or toggling of brain circuitry dynamics has also been observed by functional connectivity dynamics (Cole et al., 2014). Typical linear EEG analyses limit interpretation, since if a certain meditative technique is associated with a particular power-spectra band, explanatory power is diminished in absolute terms within the context of a highly complex, non-localized, non-linear, multi-dimensional electrophysiological framework. Although there is some value to correlating frequency domains with specific mental states, such analyses are unlikely to reflect the neurophysiological complexity accurately. 
Recent advances expanding from one-dimensional EEG correlate/s have been made, e.g. Shaw \& Routray, 2018, utilizing multivariate autoregressive MVAR modelling. Furthermore, various studies have decomposed the EEG signal into time-frequency-spatial dimensions, via electromagnetic tomography (source-localized LORETA). No consensus picture has formed, since these studies examined differing hypotheses, regions of interest (ROIs), and current density changes in diverging frequency bands, convoluted by differing practices, i.e. Mahamudra/Dzogchen (Schoenberg et al., 2018), Zazen (Faber et al., 2015), and a mixed sample comprising Tibetan Buddhists, QiGong, yoga, and Zen practitioners (Lehmann et al., 2012), entailing discrete meditation trainings/processes and mind-states. LORETA poses methodological challenges since it represents an estimation of neuronal origins of brain activity. Computational neuroscience trends aiming to model EEG correlates of meditation report decreased global alpha activity associated with increased connectivity between neurons in cortical-corticothalamic regions alongside decreased inhibitory connectivity with cells in the thalamic reticular nucleus (TRN) and secondary relay nuclei (SRN) (Saggar et al., 2015). Such observations were associated with increased stability of the mean-field model - reflecting EEG dynamics and advancement in practitioners. This reflects a viable approach to utilizing computational models to elaborate on the common neurobiological observations (e.g. decreases in alpha activity) in meditators representing sensory processing, attention, and arousal, related to the FA-OM dual-band model. Yet, interpretative limitations remain given the emphasis on mathematical representations specific to cortico-cortical, cortico-thalamo-cortical, and intrathalamic dynamics. Another study circumvented estimation/modelling issues of both approaches by investigating synchronous interactions between multiple cortical networks via simultaneous EEG-fMRI to disentangle spatial and temporal dynamics represented by Default-Mode Network (DMN) microstates (Panda et al., 2016). This study aimed to capture neural activity fluctuations related to attentional stability, yielding decreased activity in posterior cingulate connectivity concurrent to increased middle frontal temporal connectivity. Utilizing multi-modal fusion methods will contribute towards illuminating neural generators of scalp EEG-biomarkers across meditative practice. In sum, all these preceding studies disentangling spatial information from the EEG signal generally aligned with meditation-induced brain changes in morphometric and functional neuroimaging meditation studies (see Fox et al., 2014; 2016).

\section{Advances in the Classification of Practices towards Neural Marker Specificity}

A prime barrier to ascertaining systematic and replicable EEG correlates of meditative states/outcomes pertains to the absence of a clear classification system that is unifying across specific lineages/traditions/cultures. Extant EEG studies aiming to identify meditation correlates have relied almost exclusively on the FA-OM framework. Whilst this dual-band framework has been advantageous as initial reference for the neurophysiological mapping of meditation, the contemplative neuroscience field 
continues to draw from a rather narrow conceptual and methodological framework and operationalization of meditation, thus restricting inferences regarding neurobiological substrates, entailing the following ramifications: (1) EEG investigations into meditation primarily focus on attention regulation, since FA and $\mathrm{OM}$ essentially pertain to attentional processes/outcomes, representing one dimension of meditative practice and brain interaction, (2) different forms of meditation, arguably with discrete neurophysiological mechanisms/outcomes, are not clearly rationalized and defined empirically. The methodological need for scientific reports to clearly/comprehensively describe the meditative practice/training studied has been emphasized (Davidson \& Kasniak, 2015). Yet, no typology that is consistent across contemplative traditions has materialized. Furthermore, body-oriented practices (e.g. yoga, tai-chi) diffuse into reports of "meditation". A common Western misconception is that meditation is largely equated with "relaxation", which diverges considerably from meditation as rigorous mind training. Traditionally, body-oriented practices (like yoga) were preparatory for sitting meditation. Indeed, (3) studies of meditative states do not account for meditative stages that may have developed through mind-body-environment interplays at neuro-visceral or psychological level/s. (4) Confusion remains regarding the macro- and micro-levels supporting many meditative practices, i.e. disentangling meditative states (e.g. FA and OM) from meditative properties/qualities (e.g. stability, clarity), that will likely entail discrete EEG features. Finally, (5) all the preceding points (1-4) provide a generally limited view on the complexity and phenomenology associated with the development of the mind through many contemporary and historical paths of meditation. Future fruitful trajectories in contemplative neuroscience require novel methodological tools to reflect the complexity of mind-body-brain dynamics, including self-other relations and specific developmental outcomes pertaining to the transformative potential of intentional mental training/s to be included as part of the equation for data analysis/interpretation. Previous work has been helpful, yet a comprehensive integration of $1^{\text {st }}$ and $3^{\text {rd }}$ person observations called upon by neurophenomenologists remains incomplete. Furthermore, individually-specified developmental stages of progress and associated outcomes have yet to be properly integrated at various levels of experimentation.

Offering one overarching solution, we suggest an integrative developmental progressive-stage model (see Fig. 1) by which meditative practices can be conceptualized, operationalized, and quantified, with the aim to move the field forward and attain some specificity and ontological validity towards identifying replicable EEG correlates independent from differences in specific traditions/techniques. The model aims to quantify uniform stage-like developmental shifts in practice across meditation styles/techniques/lineages/cultures. Such a classification system can be utilized by researchers to categorize meditation practices and states (macro-level, front-face of pyramid), phenomenological experience, and associated properties within the practice/states (micro-level, dimensions of pyramid). 
Fig.1. Towards a Developmental-Stage Model of Meditation Classification

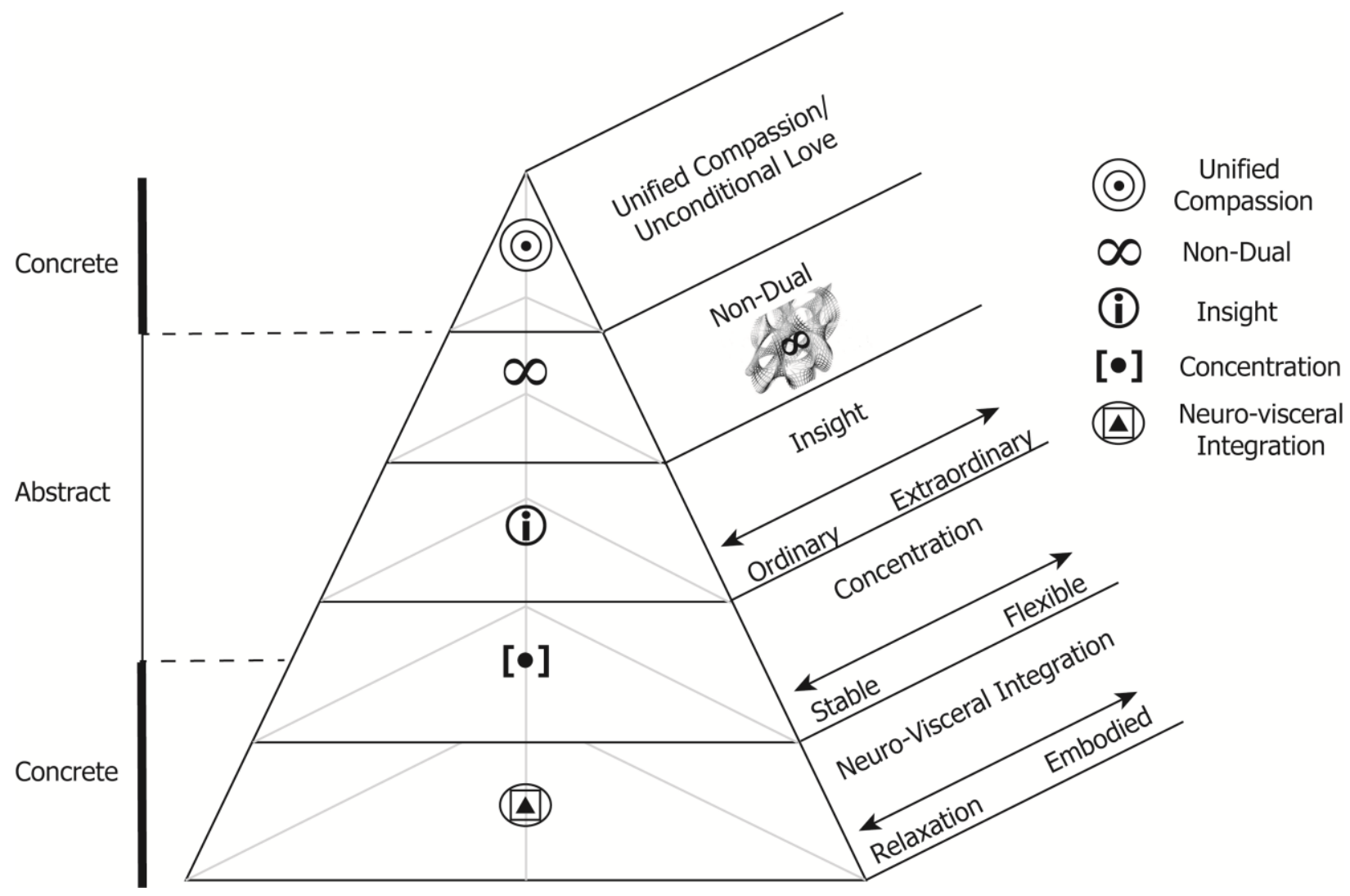

Although proposals to quantify developmental stages of human cognition (Piaget), learning/motivational needs (Maslow/McClelland), morality (Kolberg), "spirituality" (Fowler), and "ego" (Wilbur), have pragmatic value, no framework has yet conceptualized stages of development in meditative practice across traditions. Here, we propose a progressive stage-model as reference for research identifying physiological correlates of meditative practice, with scope for prescriptive purposes, mapping meditative progress, and therapeutic targets for precision medicine, and which accounts for both changes in mindbody-brain-behavior and moral-ethical development. To account for individually-specified progress of a practitioner that integrates phenomenological and cognitive-emotional perspectives, we purport internalized meditative "techniques" that facilitate development of discrete mind-states with distinct phenomenological properties; (1) "relaxation practices", characterized by neuro-visceral processes aimed to stabilize mind-body integration for more rigorous mind-oriented meditative practice. Such body-based practices integrate afferent somatosensory input, neural activity, and efferent motor output along a developmental continuum from increased parasympathetic outflow towards embodied experience, e.g. traditional yoga, tai-chi (see Gard et al., 2014). (2) “concentration practices”, characterized by training the 
mind to stabilize with phenomenal clarity in fixed-point and/or diffuse attentional scope (N.B. the FAOM dual-band concept is encompassed within this tier), facilitating flexibility for toggling between objects of engagement whilst also maintaining stable meta-awareness (Vago \& Zeidan, 2016). Shifts from concrete to abstract dimensions of experience ensue with advanced progress (advanced concentration up to non-dual states/stages are primarily abstract in phenomenological experience) ; (3) "insight practices", characterized by 'ordinary insight' that examine how the mind constructs experience, including the sense of self, external reality, sense of time, etc, leading to the overarching insight that all such represent phenomenological objects that are essentially 'empty' (illusory, construction of mind) upon arising ("emptiness"/ the "void"); (4) "non-dual practices", characterized by 'extraordinary insight' examining the "natural state" (pure essence) of mind, shifting out of a 'binary-axis' consciousness of reality, to one that is non-dual in its relation/understanding of Ultimate Reality. Non-dual states are non-referential, "selfless", non-conceptual, non-preferential, leading to a fundamental understanding of unification (note this follows the dissolution of 'self', time, and external reality, via ordinary insight practice: Schoenberg \& Barendregt, 2016; Yaden et al., 2017); (5) the "crossing over" from formal practice to one's everyday consciousness from that of a self-referential individual perspective to one of unified compassion that deeply understands the nature of the mind (constructive, recursive, pattern-generating, meaning-making) and Ultimate Reality. Unified compassion reflects the cumulative practice and integration from the preceding tiers (neuro-integrative->concentration->insight->non-dual) representing an ultimate trait-like base of operation that emanates from an inherent/experiential understanding of the futility of self-other duality and the unchanging permanent sense of "self" in place of an experience of pure interconnectedness and unity (non-duality). This is distinct from concepts of "self-compassion" advocated in secular meditation practices and concepts emphasizing social cognition/shared experience as empathy and sympathy. Additionally, the model y-axis refers to concrete/abstract experiential dimensions related to specific tiers of meditative practice/technique. Unified compassion is embodied/experienced as a concrete 'way of being' that transmutes from the abstract plane of non-dual insight.

\section{Electrophysiological Trajectories: Mathematical Multi-Dimensionality}

Focusing on one-dimension of the EEG signal to draw interpretations/conclusions regarding meditation training is problematic since it does not theoretically align with a complex, integrated understanding of the brain, and associated brain-mind mechanism/s. Evoked EEG activity represents a linear phase-locked measure to a stimulus, wherein nonlinear dynamics and mechanisms govern emergent phenomena in complex systems. Thus, mental phenomena likely emanate from self-organized, spontaneous, patterngenerating activity of the brain, and not simply from stimulus-driven processing (Fazelpour \& Thompson, 2015). Drawing solely from information provided by evoked EEG activity is analogous to estimating the 
surface area and function of a tardis ${ }^{1}$ based on its outside dimensions and appearance. Ergo, the trajectory for future EEG correlate research would be enriched within the view of 'multi-dimensionality', in terms of working mechanisms, neurophenomenological pathways, methodologies, and clinical implications. The concept that the brain is multi-dimensional has been infiltrating the wider neuroscientific purview. Recent proposals suggest investigating meditation EEG-correlates with nonlinear methods encompassing induced (non-phase-locked) signal processing techniques that may disentangle emergent properties of meditation (including meditation-based clinical outcomes/mechanisms) within the paradigm that the brain represents an open highly complex non-linear dynamical system (Schoenberg et al., 2014). Furthermore, the premise that the working mechanisms of mindfulness (as one example of meditation practice) are multi-dimensional (Schoenberg \& Speckens, 2014, 2015; Lutz et al., 2016; Zeidan \& Vago, 2016; Blanke $\&$ Brose, 2017) are accruing, in addition to taking a multi-levelled neural pathway approach for complex mind states, such as unified non-dual compassion (Schoenberg et al., 2018), and very subtle mind states during "emptiness insight practices" (Schoenberg \& Barendregt, 2016; Hinterberger et al., 2014). Nonlinear statistics, such as Lyapunov stability theory and stochastic analysis (Wang et al., 2018), and nonlinear interdependence to reflect dynamical systems complexity (Lo et al., 2018), are being embraced to investigate the poly-dimensionality and nonlinearity of meditation EEG-substrates. Fractal dimension/FD (the degree of chaos within the brain's time series) and permutation entropy (the degree of uncertainty within a random variable, such as EEG signal) may provide statistical sensitivity to detect meditation-associated neural correlates, since increased FD (network complexity) has been observed in experienced Vipassana meditators (Kakumanu et al., 2018), and "focused breath" vs. "open monitoring" meditation, significantly so for the former (Vyšata et al., 2014). Moreover, permutation entropy may have utility in gauging meditation stability, since reduced PE related to either meditative-state and/or proficiency was reported in both studies.

Topology (mathematics of continuity) represents another framework by which to shift the EEG marker paradigm. We suggest that topological mathematics also connects with implications for holographic concepts (Burch \& Di Falco, 2018) of brain mechanism and function, towards elucidating the brainsystem outside the established quantum paradigm and understanding brain-mind interplays in terms of complex open-systems within a hyperspace string theory (Gaberdiel, 2018) framework. Brain science already embraces the utility of a 4D space "hypersphere torus" to understand brain function (Tozzi \& Peters, 2016). This concept suggests that complex mental states and functions (e.g. the ability to connect

\footnotetext{
${ }^{1}$ TARDIS: Traversable Acausal Retrograde Domain in Space-Time (Tippett \& Tsang, 2017) describing a "vehicle" or "bubble" that traverses the space-time manifold, based on the theory that rather than viewing the Universe as $3 \mathrm{D}+\mathrm{a} 4^{\text {th }}$ dimension of Time, these dimensions must be imagined as instantaneous and concurrent (popularly depicted as a 'time machine' in sciencefiction classics such as "Doctor Who").
} 
past, present, future events in a single coherent mental kaleidoscope) are embedded in an "imperceptible" fourth spatial dimension of brain, which can be quantified via Borsuk-Ulam Theorem (BUT) and applied to intrinsic, low frequency, spontaneous oscillatory activity (Tozzi \& Peters, 2016), i.e. resting states network dynamics, opposed to task-evoked activity. These trends suggest that the optimal experimental design involves decomposing spontaneous brain signals into co-variation patterns providing feature vectors in $4 \mathrm{D}$ hypersphere torus space during EEG rest vs. meditation state/s. In this vein, recent computational applications utilizing algebraic topology (the mathematics describing networks with nonlocal/zero-phase/non-linear spaces/structures) identified 11 dimensions of brain (Reimann et al., 2017). Such findings shed light on synaptic transmission, the underlying mechanisms of EEG signals, whereby graphical networks relaying information flow are constructed, revealing incredibly intricate topological connectivity in local neuronal assemblies within the neocortex, whereby input/stimuli are processed by binding neurons into "cliques" of increasingly higher dimension (the more neurons within a clique, the higher the dimensions), as a specific class of cell assemblies, or "cavities" (high-dimensional holes) that guide correlated activity (Reimann et al., 2017).

The promising trajectory for a poly-dimensional approach to examining EEG substrates of meditation seems bound to a multiverse paradigm of the brain and its function, in alignment with a conceptual and methodological ontological classification system (initiated here) describing macro- and micro-level properties of discrete progressive-stage mind training/s and states. 


\section{REFERENCES}

Amihai, I., Kozhevikov, M. (2014). Arousal vs. relaxation: a comparison of the neurophysiological and cognitive correlates of Vajrayana and Theravada meditative practices. PLoS ONE, 9(7), e102990.

*Blanke, E.S., Brose, A. (2017). Mindfulness in daily life: a multidimensional approach. Mindfulness, 8(3), 737-750.

Multilevel confirmatory factor analysis was conducted to corroborate dimensions of state mindfulness with trait mindfulness self-report measures. The authors propose a 3-factor model for state mindfulness assessment comprising the (a) acting with awareness, (b) nonjudgmental acceptance, and (c) present-moment attention, domains. We suggest this proposal is pertinent in providing state-based phenomenological sensitivity when used in conjunction with EEG data for refining neuro-phenomenological approaches.

Burch, J. Di Falco, A. (2018). Surface topology specific metasurface holograms. ACS Photonics, 5(5), 1762-1766.

Cahn, R.B., Polich, J. (2013). Meditation states and traits: EEG, ERP, and neuroimaging studies. In Psychology of Consciousness: theory, Research, and Practice, Vol.1(S), pp. 48-96.

Cheng, K.S., Chang, Y.F., Han, R.P., Lee, P.F. (2017). Enhanced conflict monitoring via a short-duration, video-assisted deep breathing in healthy young adults: an event-related potential approach through the Go/NoGo paradigm. PeerJ, 5, e3857.

Cohen, M.X. (2017). Where does EEG come from and what does it mean? Trends in Neuroscience, 40(4), 208-218.

Cole, M.W., Repovs, G., Anticevic, A. (2014). The frontoparietal control system: a central role in mental health. The Neuroscientist: a review journal bringing neurobiology, neurology, and psychiatry, 20(6), 652-664.

Davidson, R.J., Kasniak, A.W. (2015). Conceptual and methodological issues in research on mindfulness and meditation. American Psychologist, 70(7), 581-592.

DeLosAngeles, D., Williams, G., Burston, J., Fitzgibbon, S.P., Lewis, T.W., Grummett, T.S., Clark, R.C., Pope, K.J., Willoughby, J.O. (2016). Electroencephalographic correlates of states of concentrative meditation. International Journal of Psychophysiology, 110, 27-39.

Fazelpour, S., Thompson, E. (2015). The Kantian brain: brain dynamics from a neurophenomenological perspective. Current Opinion in Neurobiology, 31, 223-229.

Fox, K.C., Dixon, M.L., Nijerboer, S., Girn, M., Floman, J.L., Lifshitz, M.,...Christoff, K. (2016). Functional neuroanatomy of meditation: a review and meta-analysis of 78 functional neuroimaging investigations. Neuroscience \& BioBehavioral Reviews, 65, 2018-228.

Fox, K.C.R., Nijeboer, S., Dixon, M.L., Floman, J.L., Ellamil, M., Rumak, S.P., Sedlmeier, P., Christoff, K. (2014). Is meditation associated with altered brain structure? A systematic review and meta-analysis of 
morphometric neuroimaging in meditation practitioners. Neuroscience \& Biobehavioral Reviews, 43, 4873.

Gaberdiel, M.R. (2018). String theory and spacetime geometry. Beyond Einstein, pp. 475-490.

Gard, T., Noggle, J., Park, C., Vago, D.R., Wilson, A. (2014). Potential self-regulatory mechanisms of yoga for psychological health. Frontiers in Human Neuroscience, 8, 770. doi:

10.3389.fnhum.2014.00770.

Harne, B.P., Hiwale, A.S. (2018). EEG spectral analysis on OM mantra meditation: a pilot study. Applied Psychophysiology and Biofeedback, 43, 123-129.

Hinterberger, T., Schmidt, S., Kamei, T., Walach, H. (2014). Decreased electrophysiological activity represents the conscious state of emptiness in meditation. Frontiers in Psychology, 5, Article 99. doi: 10.3389.fpsyg.2014.00099.

**Kakumau, R.J., Nair, A.K., Venugopal, R., Sasidharan, A., Ghosh, P.K., John, J.P., Mehrotra, S., Panth, R., Kutty, B.M. (2018). Dissociating meditation proficiency and experience dependent EEG changes during traditional Vipassana meditation practice. Biological Psychology, 135, 65-75.

Nonlinear statistical analyses comprising Permutation entropy (PE) and Higuchi fractal dimension (HD) that disentangled low from high proficiency in Vipassana practitioners as represented by increased network complexity. Linear spectral analyses revealed high proficiency in practice was associated in trait increases within theta-alpha $(6-10 \mathrm{~Hz})$ and low-gamma $(30-40 \mathrm{~Hz})$ bands, consistent with previous frequency-domain findings.

Kaur, C., Singh, P. (2015). EEG derived neuronal dynamics during meditation: progress and challenges. Advances in Preventive Medicine, Article ID: 614723.

Korde, K.S., Paikrao, P.L. (2018). Analysis of EEG signals and biomedical changes due to meditation on brain: a review. International Research Journal of Engineering and Technology, 05(1), 603-606.

*Lee, D.J., Kulubya, E., Goldin, P., Goodarzi, A., Girgis, F. (2018). Review of the neural oscillations underlying meditation. Frontiers in Neuroscience, 12:178. doi: 10.3389/fnins.2018.00178.

Recent overview of patterns in EEG oscillatory activity associated with meditation that highlights both some consistencies and inconsistencies/lack of clarity within the field. The authors stratify review findings within the FA-OM dual-band framework, which as we outline in this chapter, may yield limited interpretations pertaining to EEG-correlates.

**Lo, P-C, Tian, W.J.M, Liu, F-L. (2017). Macrostate and microstate of EEG spatio-temporal nonlinear dynamics in Zen meditation. Journal of Behavioral and Brain Science, 7, 705-721.

Novel approach to investigating micro-state (i.e. instantaneous states representing nonlinear neuronal dynamics with fixed parameters and specific spatial distribution, at the millisecond scale), and macro-state (i.e. statistical integration of the probability distribution of all possible microstates representing interregional neural activity in relation to a given time and function) nonlinear dynamics associated with Zen meditation. Preliminary data supports Zen meditation is 
characterized by relatively stationary and increased interconnectivity (compared to resting EEG) in frontal-midline neural oscillators.

Lutz, A., Jha, A.P., Dunne, J.D., Saron, C.D. (2016). Investigating the phenomenological matrix of mindfulness-related practices from a neurocognitive perspective. American Psychologist, 70(7), 632-658.

Nash, J.D., Newberg, A. (2013). Toward a unifying taxonomy and definition for meditation. Frontiers in Psychology, 4, Article 806.

Panda, R., B., Bharath, R.D., Upadhyay, N., Mangalore, S., Chennu, S., Rao, S.L. (2016). Temporal dynamics of the default mode network characterize meditation-induced alterations in consciousness. Frontiers in Human Neuroscience, 10, Article 372. doi: 10.3389.fnhum.2016.00372.

Quaglia, J.T., Goodman, R.J., Brown, K.W. (2016). Trait mindfulness predicts efficient top-down attention to and discrimination of facial expressions. Journal of Personality, 84(3), 393-404.

**Reimann, M.W., Nolte, M., Scolamiero, M., Turner, K., Perin, R., Chindemi, G., Dlotko, P., Levi, R., Hess, K., Markram, H. (2017). Cliques of neurons bound into cavities provide a missing link between structure and function. Frontiers in Computational Neuroscience, 11, Article 48. doi:

10.3389.fncom.2017.00048.

Pioneering article outlining the utility of mathematical topology in elucidating complex synaptic connectivity, that provides an empirical and quantifiable link between neural network structure and emergent function. Findings revealed intricate neocortical networks comprising abundant cliques of neurons bound into cavities that guide emergent activity and processing. We suggest such approaches are highly pertinent in moving the contemplative neuroscience field towards multidimensional investigations of meditation-associated brain states/processes so to identify sophisticated replicable EEG-correlates gauging more subtle phenomenology/processing during meditation.

Saggar, M., Zanesco, A.P., King, B.G., Bridwell, D.A., MacLean, K.A., Aichele, S.R., Jacobs, T.L., Wallace, A.B., Saron, C.D., Miikkulainen, R. (2015). Mean-field thalamocortical modelling of longitudinal EG acquired during intensive meditation training. Neuroimage, 114, 88-104.

Sanger, K.L., Dorjee, D. (2016). Mindfulness training with adolescents enhances metacognition and the inhibition of irrelevant stimuli: evidence from event-related brain potentials. Trends in Neuroscience and Education, 5(1), 1-11.

Schoenberg, P.L.A., Ruf, A., Churchill, J., Brown, D.P., Brewer, J.A. (2018). Mapping complex mind states: EEG neural substrates of meditative unified compassionate awareness. Consciousness and Cognition, 57, 41-53.

*Schoenberg, P.L.A., Barendregt, H.P. (2016). Mindful disintegration and the decomposition of self in healthy populations: conception and preliminary study. Psychological Studies, 61(4), 307-320.

Non-phase-locked event-related (de-)synchronization (ERD/ERS) provided the first nonlinear EEG examination of advanced stage Vipassana culminating in phenomenological clarity and stability of the "emptiness" of all that arises (Insight tier on the included development-stage model within this 
chapter). The N400 event-related potential (ERP), or 'meaning waveform' was also extracted, wherein reduced amplitude was observed to stimuli (i.e. perceptually, stimuli became less 'meaningful'/salient) following intense experiences of the "void" associated with the insight process. Moreover, this study provided an initial postulation in terms of an 'equanimity neural marker' in the form of modulated beta-synchrony and beta-rebounding, representing cortical stabilization (cortical buffer) during the transformative 'disintegrative' process. [* For academic transparency, this publication was within the peer-review system for 2-years, originally submitted in 2014].

Schoenberg, P.L.A., Speckens, A.E.M. (2015). Multi-dimensional modulations of $\alpha$ and $\gamma$ cortical dynamics following mindfulness-based cognitive therapy in Major Depressive Disorder. Cognitive Neurodynamics, 9, 13-29.

Schoenberg, P.L.A., Speckens, A.E.M. (2014). Modulation of induced frontocentral theta (Fm- $\theta$ ) eventrelated (de-)synchronization dynamics following mindfulness-based cognitive therapy in Major Depressive Disorder. Cognitive Neurodynamics, 8, 373-388.

Schoenberg, P.L.A., Hepark, S., Kan, C.C., Barendregt, H.P., Buitelaar, J.K., Speckens, A.E.M. (2014). Effects of mindfulness-based cognitive therapy on neurophysiological correlates of performance monitoring in adult attention-deficit/hyperactivity disorder. Clinical Neurophysiology, 125, 1407-1416.

Shaw, L., Routray, A. (2018). Topographical assessment of neurocortical connectivity by using directed transfer function and partial directed coherence during meditation. Cognitive Processing, doi.10.1007/s10339-018-0869-2 [Epub ahead of print].

Tippett, B.K., Tsang, D. (2017). Traversable acausal retrograde domains in spacetime. Classical and Quantum Gravity, 34, 095006.

Tozzi, A., Peters, J.F. (2016). Towards a fourth spatial dimension of brain activity. Cognitive Neurodynamics, 10, 189-199.

Vago, D.R., Zeidan, F. (2016). The brain on silent: mind wandering, mindful awareness, and states of tranquility. Annals of the New York Academy of Sciences, 1373, 96-113.

Vyšata, O., Schätz, M., Kopal, J.,Burian, J., Procházka, A., Jiří, K., Hort, J.,Vališ, M. (2014). Non-linear EEG measures in meditation. Journal Biomedical Science and Engineering, 7, 731-738.

Wang, X., Jiang, G-P, Wu, X. (2018). Outer synchronization for general weighted complex dynamical networks considering incomplete measurements of transmitted information. Complex Networks IX, pp.139-150.

Yaden, D.B., Haidt, J., Hood, R.W., Vago, D.R., Newberg, A.B. (2017). The varieties of SelfTranscendent Experience. Review of General Psychology, 21(2), 143-160.

Zeidan, F., Vago, D.R. (2016). Mindfulness meditation-based pain relief: a mechanistic account. Annals of the New York Academy of Science, 1373(1), 114-127. 


\section{GLOSSARY OF TERMS}

Macros-scale States of Meditation Terminology (relating to pyramid stage-model):

\begin{tabular}{|c|c|}
\hline $\begin{array}{l}\text { Ordinary } \\
\text { Insight: }\end{array}$ & $\begin{array}{l}\text { The process of decomposing 'objects' of mind to their constituent units, so to fundamentally understand } \\
\text { the mind-level construction of sense of relative reality, experience of "self", experience of time, pattern- } \\
\text { forming, meaning-making, and so on. Ordinary insight pertains to the practitioner understanding the } \\
\text { formations/structure/parameters of their own mind at micro-to-macro levels that provides the foundation } \\
\text { for extraordinary insight. It also forms the basis for gross-level "emptiness" (see below) practice; i.e. the } \\
\text { emptiness of the objects of mind as they come and go. }\end{array}$ \\
\hline $\begin{array}{l}\text { Extraordinary } \\
\text { Insight: }\end{array}$ & $\begin{array}{l}\text { Awareness itself is the focus of observation. From this perspective of observation, the realization } \\
\text { (insight) that the mind and its manifestations are essentially inseparable, as with a dreamer and a dream. } \\
\text { This process is a key pre-requite for understanding/experiencing subtle-level "emptiness" (below) so to } \\
\text { experience dimensions of Ultimate Reality (also below). }\end{array}$ \\
\hline Emptiness: & $\begin{array}{l}\text { Realization, experience, and fundamental understanding (wisdom) of absolute truth; namely, that } \\
\text { manifestations of mind (including attachment of "self") are "empty" upon arising i.e. all that exists } \\
\text { within (internally) and through (eternally) mind is fundamentally illusory, transient, and impersonal (and } \\
\text { is a key prerequisite for 'equanimity' and experience of liberation). In its essence, emptiness emphasizes } \\
\text { the lack of inherent, independent existence of the self and all phenomena. At the very subtlest levels, the } \\
\text { experience of emptiness provides the foundation for the most arcane experience/understanding of non- } \\
\text { duality (the next tier within the progressive stage-model presented). } \\
\text { Not to be confused with 'nihilism', since nihilistic philosophies (in themselves objects of mind) question } \\
\text { the intrinsic meaning or value of existing in relative terms for the individual, and as such one could } \\
\text { argue remain highly attached to a sense of self (are ego-full). }\end{array}$ \\
\hline Non-Dual(ity): & $\begin{array}{l}\text { Complete shift out of the binary perspective/s and continuum/s of mind, also synonymous with } \\
\text { "simultaneousness"/ "instantaneousness". Non-duality does not render the mind and its } \\
\text { manifestations/objects as one and the same thing; rather, that mind and its objects, or experience and its } \\
\text { contents, always come together as one entity. Via this process/understanding/experience, dualistic } \\
\text { manifestations of mind no longer ensue, since liking/disliking, good/bad, preference/non-preference, are } \\
\text { understood to exist from the same root (the mind), and cease to exist on the Ultimate plane of Reality } \\
\text { (complete shift to absolute experience of being that is vivid, bright, vibrant, infinite, non-local, non- } \\
\text { spatial). It may be experienced as a rapidly expanding dimension of consciousness that instantaneously } \\
\text { 'implodes' into an indivisible axis of experience that is simultaneously ever-changing (expansive, } \\
\text { explosive) and static (unchanging, constant, like a photo-shot in time). N.B. timelessness, selflessness, } \\
\text { have fallen away prior during 'ordinary insight' - the realization and understanding of the workings and } \\
\text { proliferation of mind constructs. }\end{array}$ \\
\hline $\begin{array}{l}\text { Ultimate } \\
\text { Reality: }\end{array}$ & $\begin{array}{l}\text { Absolute perspective that is not determined or experienced by manifestations of the mind. Ultimate } \\
\text { Reality may be experienced as dynamically vibrating, ever-changing, fluidic, clear-light, completely } \\
\text { devoid of concept/object (not to be confused with the self-object divide, since in absolute terms, the } \\
\text { "self" is a concept/object that exists only as a relative reality of mind). }\end{array}$ \\
\hline $\begin{array}{l}\text { Unified } \\
\text { Compassion: }\end{array}$ & $\begin{array}{l}\text { Unconditional/altruistic love that is interconnected and encompassing, emanating from the } \\
\text { insight/experience of non-dual dimensions. Its highest form translates within the external world as } \\
\text { "service-to-others" (opposed to "service-to-self" orientation) contributing to the altruistic motivation } \\
\text { towards alleviation of suffering for all sentient beings (opposed to pursuing one's own illusory will that } \\
\text { inevitably afflicts suffering upon others). In Western culture this can often be at odds with acceptable } \\
\text { behavior/s, since the West values and 'rewards' (for earthly 'success') individualization and self- } \\
\text { promotion over values of kindness/compassion (that may be viewed as "weak" in secular contexts), or } \\
\text { operating from non-self-referential perspectives (that may be viewed as confusing or "strange" within } \\
\text { secular contexts). Unified compassion embodies strength and courage for the benefit of all, opposed to } \\
\text { aligning with the conditioned mind and conditioning of society for the benefit of one (ones-"self"). }\end{array}$ \\
\hline $\begin{array}{l}\text { Abstract- } \\
\text { Concrete } \\
\text { Dimension: }\end{array}$ & $\begin{array}{l}\text { The phenomenological quality within any given tier. At neuro-visceral integrative and concentrative } \\
\text { practice tiers of the model, experience is largely concrete in nature, i.e. body-centric and effable. } \\
\text { Advanced concentrative practice and the insight and non-dual stages shift into abstract planes of } \\
\text { experience that are not usual from the everyday body-centric experience of external/internal reality, and } \\
\text { may be difficult to linguistically formulate. Unified compassion shifts back to a concrete axis, since the } \\
\text { practitioner has developed through the abstract stages and now reintegrates consciousness back into a } \\
\text { concrete operation of being so to enact and radiate unified compassion in everyday life (off the cushion). }\end{array}$ \\
\hline
\end{tabular}




\section{*Note on appropriation and translation of Buddhist concepts}

It must be noted that perceptions of the terminology used within the proposed unified progression-stage model will be interpreted via one's own experience, previous readings, and preconceptions of "meditation". Many of the terms used to describe the model may bear resemblance to historically Buddhist concepts. Yet, the authors wish to emphasize that some terms are in context and others have been re-contextualized so to propose an integrative model by which researchers can map mind-brain-body observations onto the tiers and dimensions described. Indeed, there may be attempts to map existing concepts from the Buddhist literature onto the model, and yet, terms like "insight" or "non-duality" may not find correspondence as described in the Buddhadharma.

**Note on the pragmatic use of this model for means of rigorous examination of markers of meditation. Related to the note above, the authors wish to emphasize that the usage of terminology (and further clarification/description in the glossary) is; (a) to encourage contemplative neuroscientists to describe the phenomenology relating to the meditative states/stages examined in their reports, and (b) to match such phenomenology to the progression-stage model presented, so to enrich the precision of neural marker research. As one example, the authors appreciate the "non-dual" tier of the model potentially has many interpretations from the traditional terminology and concepts. For example, in the Theravadan tradition, some interpretations of 'Samadhi' have proposed this as a non-dual state. Although if we go by the phenomenological descriptions, it is in fact defined as highly stable single-pointed mind (for which we re-contextualize in this model as falling on the concentrative tier within the FA dimension). Another potential misinterpretation pertains to the Pali term, "nirodha-samāpatti" or "sañna-vedayita-niroda" which have been conflated with non-duality. In Theravadan traditions Nirodha (cessation) and Samāpatti (fruition/attainment) refer to extinguishing the self broadly speaking through the experience and realization of cessation of sensation, perception, and emotion. This realization has further been associated with insight into the arising and falling of phenomena within and through mind. This phenomenological description may be classified within the "insight" tier of the model (more specifically 'ordinary insight' that understands the emptiness of all phenomena, including the manifestations of mind and self). Another potential misinterpretation pertains to Transcendental Meditation (TM) schools who suggest that through focusing on a mantra (of which we note is an object of mind) one comes to a non-dual state. From the descriptions of this practice, the authors wish to note that TM is within the neuro-visceral tier of this proposed model, since through the iteration of the mantra (object of mind) the mind becomes lax, not sharp as with concentrative and above tiers. Within the Hindu Advaita Vedanta, mantra will occupy the field of consciousness as a tool to aid the mind to withdraw from the bombardment of external and internal sensory distraction. The Sanskrit term "pratyahara" (withdrawal of the senses) is described as a form of mental relaxation engaged through mantra to prepare the mind for progress through meditation. As this process of relaxation (both relaxation of the body and laxity of mind) can give rise to dissociative sensation/experience, such states may be misconstrued as 'non-dual'. The authors wish to emphasize that the non-dual tier within this proposed progressive-stage model encompasses meditative states and stages that are non-linear in experience, i.e. not necessary 'unification' interpreted as one-pointedness, or hyper-concentration as "stillness"/absorption, rather to emphasize the vibrancy, vividness, fluxing, non-linearity (multi-dimensionality), instantaneousness, of non-dual states and stages that are the "essence" of absolute reality and experience devoid of the conditioned mind and relative reality. Thus, it is the responsibility of the researcher to disentangle the meditative practices they are examining and map onto the phenomenological distinctions of the presented model stages, for which this glossary may provide some reference. 